\title{
Experimental Investigations into the Effects of Lithology on Acoustic Emission
}

\author{
Baozhu Tian ${ }^{1,}{ }^{*}$, Shanjun Liu ${ }^{1}$, Jian Li $^{2}$, Peng Liang ${ }^{2}$, Yanbo Zhang ${ }^{2}$ and Zhanle Wang ${ }^{3}$ \\ ${ }^{I}$ College of Resources and Civil Engineering, Northeastern University, Shenyang 110819, China \\ ${ }^{2}$ College of Mining Engineering, North China University of Science and Technology, Tangshan 063009, China \\ ${ }^{3}$ Electronic Systems Engineering, University of Regina, Regina S4S0A2, Canada
}

Received 20 July 2015; Accepted 3 November 2015

\begin{abstract}
In order to study how lithology affects acoustic emissions (AE), a series of tunnel rock burst simulation experiments, monitored by acoustic emission instruments, were conducted on granite, marble and basalt. By analyzing the characteristic parameters, this study found that $\mathrm{AE}$ events occur more frequently during the rock burst process on granite and basalt. Marble remains dormant until $75 \%$ of the loading time before the peak, at which point, cracks develop rapidly and $\mathrm{AE}$ events dramatically increase. During the rock burst process, the AE energy release demonstrates that low energy is released in the incubation phase and robust energy is released during the later phase. Before the rock burst occurs, increased in the heterogeneity index $\mathrm{C}_{v}$ values of the $\mathrm{AE}$ event are subject to lithology. The $\mathrm{C}_{v}$ values of granite and basalt have an increase of about $0.2-0.4$, while marble shows an increase of 1.0-1.2. The heterogeneity index $\mathrm{C}_{v}$ value of an $\mathrm{AE}$ event is in line with the rock burst process.
\end{abstract}

Keywords: Rock burst, Acoustic emission, Energy, Lithology, Rock

\section{Introduction}

Rock burst is a common type of dynamic disaster in underground engineering areas under high stress. During tunnel excavation or other types of underground engineering, tunnels produce concentrated areas of stress, the elastic strain energy in the rock suddenly and violently releases under certain conditions, and the phenomenon of burst, peeling, ejection and cast were shown in the process [1]. In recent years, many mines have entered depth mining with underground chamber engineering, the probability of rock burst incidents is greatly increased. The question of how best to carry out effective rock burst monitoring has became an important problem in the underground engineering field. The occurrence and development of rock bursts have certain mechanical mechanisms. Given the analysis of effect factors occurring during rock bursts, it is known that the rock failure is affected not only by internal factors in deep engineering rock masses like physical mechanics, the structure and the nature of the surrounding environment (stress, tectonic environment, hydrogeological and geothermal environment etc.), but also by external inducing factors, including deep long tunnel engineering designs, construction, mining and far field earthquakes, all of which were related to the disturbance. Considering different factors, analyzing and describing the rock burst mechanism are different matters. Due to the complexity of the influence factors in the surrounding rock and rock burst, no universal conclusion has

* E-mail address: tianbaozhu1111@sina.com ISSN: 1791-2377 @ 2015 Kavala Institute of Technology. All righte recerved yet been reached regarding the rock burst mechanism.

Acoustic emissions(AE) are caused by the force of rock crack propagation behaviors. Different types of rocks have different micro structure characteristics, and their acoustic emission levels are likewise different[2]. The rock AE level may reflect rock fissures that developed during the loading process and other rock properties as well as the rock damage characteristics influenced by the stress state. The United States Bureau of Mines applied AE technology to monitor and forecast rock bursts in 1930s; since then, the AE technique has been widely used in geotechnical and mine rock stability monitoring. $\mathrm{Xu}$ et al. established a biaxial compression condition relationship between $\mathrm{AE}$ and rock damage variables, concluding that rock burst can be forecast via acoustic emissions[3]. He et al. carried out four types of rock burst experiments on Beishan granite using different unloading rates and found that AE occurs largely in the midand low-frequency bans; as the unloading rate reduces, it varies from dense to discrete[4]. Using the rock burst $\mathrm{AE}$ time-frequency and micro fracture mechanism, Miao et al. analyzed the strain type characteristics in rock bursts[5]. Through both laboratory experiments and numerical simulation, Zhang et al. studied the influence of tectonic stress on granite tunnel rock bursts and visible image, acoustic emissions and far infrared variations, he established a multi-physics parameter early rock burst monitoring method[6]. Li et al. carried out simulations on granite rock burst in natural and saturation states, and discussed the impact of water on rock burst acoustic emission laws; in the process, she discovered that the acoustic emission event on granite in its natural state takes longer to calm down than in its saturated state[7]. Testing the failure process of $\mathrm{AE}$ on 
quartz diorite which has rock burst tendencies, Yuan et al. found that, as stress increased, the AE spectrum developed from low frequency to high frequency, and as the second frequency emerged, the AE count and energy gradually concentrated to high- frequency, which provided a basis for rock burst prediction in this type of rock[8].

Studies has shown that a large number of experiments on $\mathrm{AE}$ rock burst monitoring have been carried out, and important progress has been made[9], [10], [11]. By analyzing the $\mathrm{AE}$ information during the rock failure process, the development of internal microscopic fissures can be reversely deduced until the entire process of macroscopic damage been determined, which provides a new means for researching rock burst failure rules and mechanisms. At the same time, by capturing the AE multi-dimensional precursor information, a new method for predicting rock burst disaster is now available[12], [13], [14]. However, for rock burst lithology, different lithological rock bursts, abnormal AE characteristics and their rules in the rock burst process, a systematic and in-depth study is required.

Based on the above analysis, simulation experiments on different lithological tunnel rock bursts have been carried out, using $\mathrm{AE}$ to monitor the entire rock burst process from formation to occurrence. By analyzing AE parameters and their laws in the rock burst process under different lithological conditions, we aim to determined $\mathrm{AE}$ response characteristics in order to study rock burst mechanisms from a new perspective and provide a basis for rock burst precursory study.

\section{Methodology}

\subsection{Sample selection}

Rock burst occurs in hard, brittle igneous rocks and metamorphic rocks, including granite, gneiss, limestone, and diorite[15]. Considering rock burst cases, there have been rock bursts in the Linglong gold mine and in the Hongtoushan copper mine, marble rock bursts in the Jinping Hydropower Station, and basalt rock bursts have occurred numerous times in the White Jiao mine. Based on this, granite, marble and basalt were chosen to conduct the rock burst simulation experiments. The granite was collected from Laizhou, Shandong Province, where the main minerals are quartz, biotite, and plagioclase, all with hard textures and grey surfaces. Basalt was collected from Inner Mongolia, where main minerals are pyroxene, olivine, and biotite, all with dense textures, fine particles and black surfaces. Marble was collected from Laizhou, Shandong Province, where the main minerals are calcite and dolomite, uniform in texture, and white on the surface.

To understand the basic mechanical properties of the selected rock samples, while providing the basis for the rock burst experiment design. first of all, the rock specimens are first put through uniaxial tensile and compressive strength tests to obtain the rock' $\mathrm{s}$ basic mechanical parameters. The basic mechanical parameters of the rock samples are shown in Table 1.

Table 1.The basic mechanical parameters of specimens.

\begin{tabular}{lccccc}
\hline Parameters & $\begin{array}{c}\mathrm{E} \\
(\mathrm{GPa})\end{array}$ & $\mu$ & $\begin{array}{c}\sigma_{\mathrm{c}} \\
(\mathrm{MPa})\end{array}$ & $\begin{array}{c}\sigma_{\mathrm{t}} \\
(\mathrm{MPa})\end{array}$ & $\begin{array}{c}\mathrm{P} \\
\left(\mathrm{g} / \mathrm{cm}^{3}\right)\end{array}$ \\
\hline Granite & 4.28 & 0.24 & 110.42 & 8.17 & 2.57 \\
\hline Marble & 3.27 & 0.36 & 80.78 & 6.06 & 2.8 \\
\hline Basalt & 3.96 & 0.15 & 215.67 & 16.1 & 2.77 \\
\hline
\end{tabular}

Secondly, rock burst occurrences require a certain degree of brittle fracture; the rock itself needs to have a lot of elastic energy storage properties. Dozens of methods exist for evaluating rock burst tendencies from the lithological perspective; the authors chose the strength brittleness coefficient $(\mathrm{R})$ method to calculate the granite rock burst tendency[16]. The expression of the evaluation of rock burst tendency is shown in Formula (1).

$R=\sigma_{c} / \sigma_{t}$

The bigger $\mathrm{R}$ corresponds to greater brittleness in the rock and increased rock burst tendency; a criterion of $\mathrm{R}$ as follows; (1) $\mathrm{R}<10$ indicates no rock burst; (2) $10 \leqslant \mathrm{R}<18$ indicates moderate rock burst; (3) $R \geqslant 18$ indicates strong rock burst.

As can be seen from the experiment's measured data shown in Table 2, three types of rock burst tendencies are at the medium level. Due to fact that the elastic modulus and compressive strength both remain high, a rock sample can absorb a great deal of strain energy during loading process, while still having a strong rock burst tendency. Samples are therefore suitable to serve as the basic material in this rock burst experiment and adopting this type of rock offers more practical reasons to study the problem in this article.

Table 2.Tested result of rock burst tendency.

\begin{tabular}{|c|c|c|}
\hline Rock & Brittleness coefficient & Rock burst tendency \\
\hline Granite & 13.46 & Medium \\
\hline Marble & 13.33 & Medium \\
\hline Basalt & 13.4 & Medium \\
\hline
\end{tabular}

\subsection{Experimental methods}

In the experiment, 5 rectangular pieces of each type of rock were used to test three kinds of lithological samples with the following specifications: $150 \mathrm{~mm} \times 150 \mathrm{~mm} \times 75 \mathrm{~mm}$. Because the influence range is 3-5 times that of the tunnel radius after excavation, a hole with $30 \mathrm{~mm}$ diameter was drilled in the middle of each sample.

Two-way loading tunnel excavation was simulated; secondary stress redistribution led to rock burst damage, while the horizontal load was controlled by force, and the axial load control mode used displacement. The experimental design was loaded horizontally to $90 \mathrm{KN}$, then loaded axially to $0.3 \mathrm{~mm} / \mathrm{min}$ until rock burst occurred. The load equipment consists of an RLW-3000 servo pressure testing machine, the camera is an AVT Pike F421B and the acoustic emission instrument is a PCI-2 type that was produced by American Physical Acoustics. During the experiment, a camera was used to acquire visible photos and observe rupture generations and development. To ensure the data in each system corresponded exactly to the time, prior to the starting of the experiment, each experimental data acquisition system was timed for synchronization. The experiment process is shown in Figure 1. 


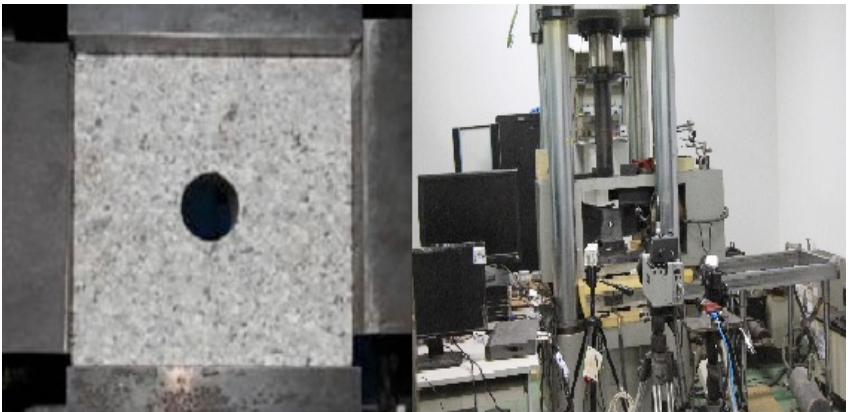

Fig. 1. The experiment process.

\section{Result}

\subsection{The rock burst characteristics of different lithological} rock

In the rock burst simulation experiment, three kinds of lithological samples occurred at different degrees of rock burst destruction. Granite, marble, basalt occurred an obvious phenomenon of rock burst ejection and exfoliation. There is no obvious phenomenon around the sample holes during early loading, when the hole remained stable. On the surface along the left and right sides of the orifice, small particles began to appear, catapulted out when the axial load reached a certain point. With continued loading, the particle ejection phenomenon appeared within the hole, and then developed into violent ejection of debris, exfoliation and a rock avalanche accompanied by the ringing sound of the burst. The rupture position extended from the orifice to the bore-hole in the axis direction along the left and right edges. Obvious cracks appeared gradually on both sides of the hole's inner wall. When the load limit was reached, there occurred around the samples' holes a large area caving in; the hole was severely deformed and rock samples suddenly lost stability and collapsed. The experimental results are consistent with the four stages: stable phase, particle ejection, exfoliative and comprehensive collapse four stages.

A number of engineering records show that rock bursts occur in different lithologies; its status or phenomenon also vary[17], [18]. Granite is intrusive igneous rock, marble is metamorphic rock, and basalt belongs to spray type lava rocks. The rock burst process changes with different lithologies.

After the granite hole appeared fine particles catapulted out, and the catapult particles gradually extended to the inner wall of the hole along the hole axis, developing into the violent ejection of debris. The violent ejection continued for some time, resulting in a deep "rock burst pit" on two sides of the wall. A smoke-like result occurred inside the hole, with a large rock powder spray; the surface of the sample around the hole has a large cave-in area. Lastly, with noise, the rock collapsed, as shown in Figure 2.
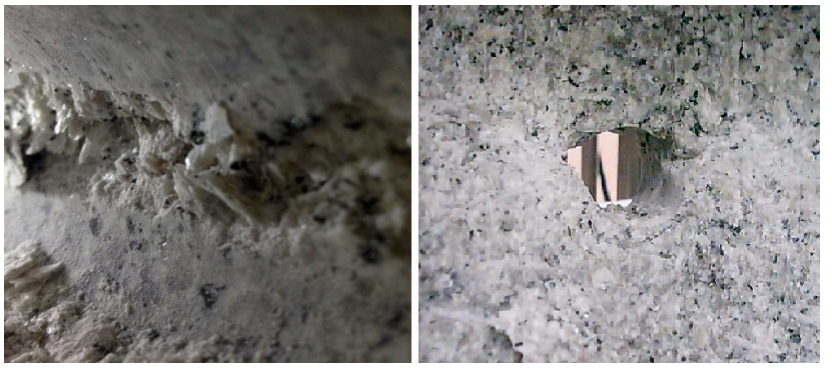

Fig. 2. Rock burst of granite.
The ejection around edge of the marble orifice appeared late. Slight particle ejection occurred, and the hole fell briefly into a calm state at almost the same time; the ejection did not spread around the hole wall, but started ejecting small pieces. Large pieces of flake-off appeared at the left and right sides of the hole walls. There was no obvious presence of mistlike conditions inside the hole. The hole wall appeared to have multiple circular cracks; finally, around the hole, a great number of splitting cracks formed upward, parallel to the maximum principal stress direction, at which point the sample lost bearing capacity. Compared with the granite, the marble rock took less time for ejection and exfoliation during the rock burst process. The broken form is shown in Figure 3.

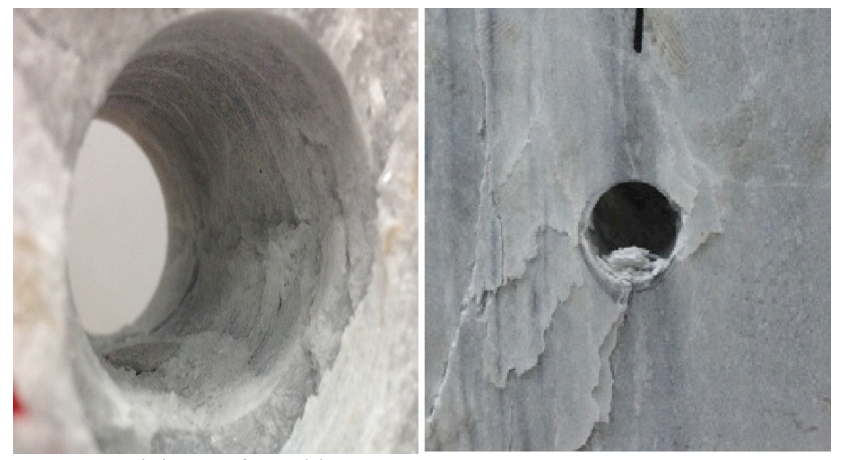

Fig.3. Rock burst of marble.

Compared with marble, the basalt rock burst had a more robust occurrence. It took a short time to extend to the inner wall after the first ejection of rock pieces around the hole. Cut-through cracks instantly appeared on the left and right sides of inner wall, at which point pieces of rock ejected outward suddenly and violently. The time spent during this violent ejection was longer than it occurred in the granite, but less time than it occurred in the marble; a smoke-like appearance spread over the sample, with a loud pop sound, pieces were rapidly ejected a distance away. A large area collapsed on the surface, and splitting cracks appeared around the hole, parallel with the direction of the maximum principal stress, as shown in Figure 4.

A summary analysis found that the granite rock burst was the most dramatic, followed by basalt, while marble had the lightest response. This has a close relationship with the peak strength and elastic modulus of the rock. In the rock burst simulation experiment, three rock samples were eventually destroyed at different peak intensities. The higher the peak intensity is, the harder the rock is, and the greater its ability to absorb the surrounding force. The elastic modulus of rock relates to its energy storage capacity; the greater its values, the greater the stress it takes to perform elastic deformation and the stronger the rock's energy storage capacity is [19], Basalt and granite's peak strengths and elastic modulus are higher, and a larger amount of energy is stored during their rock burst phase; when rock burst occurs, more energy is released and more of it is changed into the rock's kinetic energy. Which is what cause violent ejections inside the sample hole. Marble's ability to store energy is relatively weak, thus the clastic rock burst ejection was not strong during the process. 


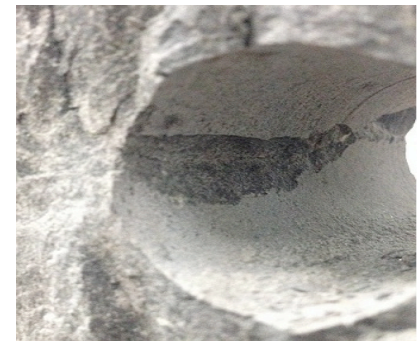

Fig.4. Rock burst of basalt.

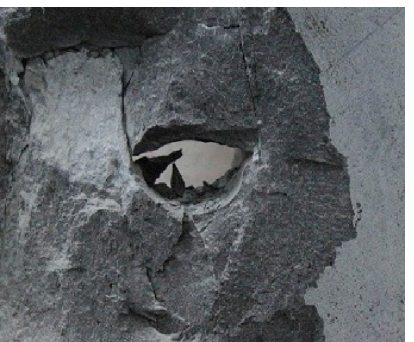

\subsection{Acoustic emission characteristics of rock burst in different lithologies}

\subsubsection{Characteristic of acoustic emission event in the rock burst process.}

Cracks generated in the internal area of the rock under high stress conditions can lead to acoustic emissions, whether under microscopic dislocations or under macroscopic twinning actions and intergranular slips or by passing through or along the mineral crystal fracture propagation phenomena. An acoustic emission event may reflect the frequency of the acoustic emission source activities. Figure 5 is a rock burst curve with axial loads -an-acoustic emission event: due to space constrains, only the times of granite, basalt and marble will be stated here.

As seen from Figure 5, during the bursts in the three rocks, the acoustic emission events are different for each changing feature. The granites initial stage of acoustic emission activity is more active; during the rock burst the periodic acoustic emission is obvious, in initial zone I, violent region II, drop zone III and quiet area (quiet zone) IV, as shown in Figure 5 (a). Acoustic emissions occur largely during the load to the peak period $10 \%$ to $80 \%$ of the time, and they continue to fluctuate. Initial rock samples showed earlier acoustic emission, high bulk acoustic emission frequency characteristics, which have some contact with the material composition and internal structure of the granite. The granite rock sample was composed of feldspar, quartz, hornblende and other minerals. Because of granite's compression strength, the inhomogeneity of particles and because the natural defects in the internal structure were different, granite was sensitive to stress, and its cracks propagated dynamically under loading, which was performed as rich acoustic emission activities.

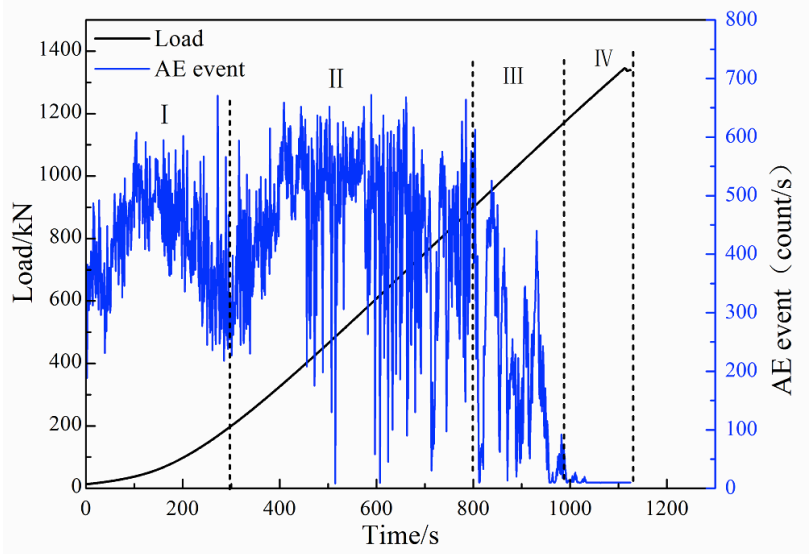

(a)

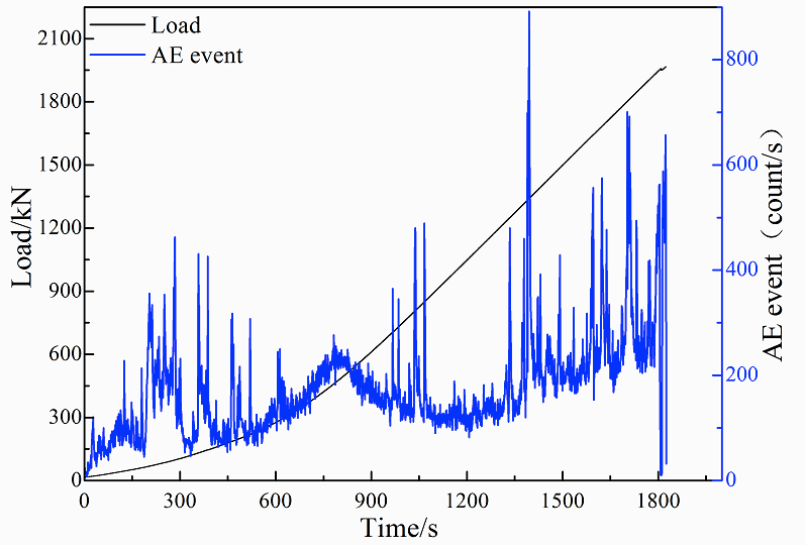

(b)

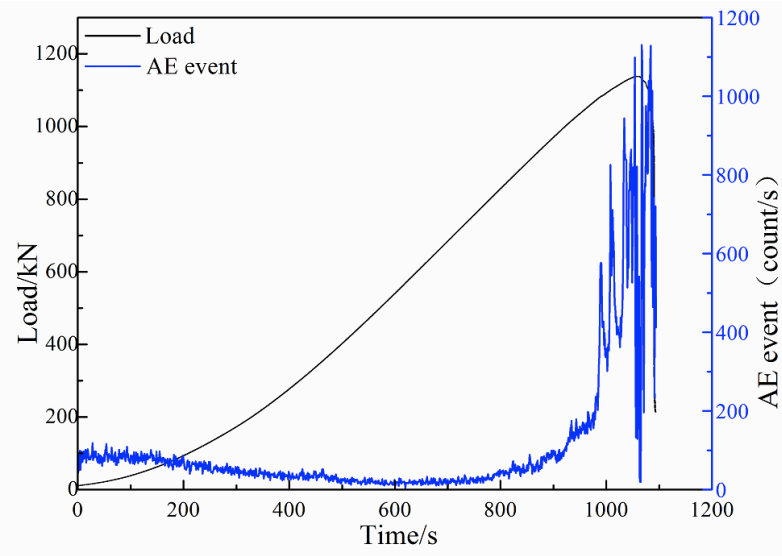

(c)

Fig.5. Curves of load and AE event. (a) Granite.(b) Basalt.(c) Marble

The acoustic emission activity was more active in basalt during the entire process, and existed from the initial stage of rock burst. As the loads increased, the acoustic emissions were more increasingly active and the acoustic emission activity remained active until the sample, reached the peak load and the main rupture occurred, as shown in Figure 5 (b). The basalt sample was composed primarily of a mafic composition of feldspar and pyroxene, containing small amounts of olivine, hornblende, biotite and other minerals; because it featured uneven crystallized connection, it cracked downward easily and was extended by force, so the acoustic emissions were more active. When the inner wall of the basalt rock burst, the ejection holes extended faster, and the crack propagation speed was also faster, so the latter part of the acoustic emission event was more active than the initial stage.

The initial stage of the marble's acoustic emission activity was relatively calm; $\mathrm{AE}$ events were rare, from about $75 \%$ of the loading time until the peak, the acoustic emissions were dormant, as shown in Figure 5(C). Close to the peak load, the rate of acoustic emission events sharply increased and reached a higher level. The marble' $s$ rate of acoustic emission event was higher than granite and basalt. The marble sample was mainly composed of calcite and dolomite, the main chemical composition of which is carbonate. The mineral composition is simple, so the initial stress effects were not sensitive, and the marble showed a longer quiet $\mathrm{AE}$ period. Close to the main rupture, cracks rapidly expanded to the cavity wall, and the particle ejection phenomenon emerged, which showed that obvious acoustic emission events appeared late. Many ejections developed simultaneously along the inner wall; cracks spread out and 
cut through rapidly, at which point the rate of the acoustic emission event quickly rose to a higher level.

The foregoing analysis shows that the acoustic emission activity was more active the granite and basalt rock bursts and the high acoustic emission event occurred in the initial stage. The initial stages for granite had the highest event, about 500 times/s, followed by basalt, 300-400 times/s, while marble was at a minimum of 30 to 90 times/s, which relates closely to the formation of the different rock samples. Granite and basalt are igneous rocks, formed by the condensation process of crustal magma intrusions or surface spray; magma flows change with temperature, pressure and other conditions. Its chemical composition, structure and composition of mineral constitutes are complex, so the magma connection between the rock particles of crystalline varies; the cracks are active under stress, and acoustic emission activity is more abundant. However marble is a metamorphic rock formed under the Earth's internal forces, because it is composed of a simple composition of carbonate minerals, its mineral particles join more uniformly, and are therefore not sensitive to stress. The crack growth in the initial stage was less, so the initial acoustic emission event was lower.

\subsubsection{The process of rock burst characteristics of acoustic emission energy}

The rock loaded failure process is essentially an energy conversion process of absorbing and releasing. From the perspective of acoustic emission energy, the variation characteristics during the process of rock burst test are more typical. AE energy represents how much elastic strain energy has been released during the rock burst process in the unit time, and reflects the amount of energy released in the burst. The rock samples show the acoustic emission characteristics to be initially quiet, but growing more concentrated upon release, reflecting the burstiness and instantaneity of rock burst.

Fig. 6 presents three kinds of loads for the rock sample acoustic energy emission -time curve. Over $80 \%$ of the time during the previous peak load, the three kinds of rock showed almost no energy being released: this indicates the rock burst's energy-storage phase. Part of the external force is stored in the form of elastic deformation; another part is consumed by the internal mechanism of nonlinear deformation. Then the rock burst energy is released at a high speed over a short time. Stored elastic energy is then converted into cracks initiated by surface energy and the release of kinetic energy via rock ejection and thermal energy and so on, characterized by the rapid increase in $\mathrm{AE}$ energy. This robust energy release is the direct driving force of the rock burst.

Different lithology is one of the important factors affecting rock burst energy release. Three kinds of rock energies are released at different magnitudes. Through these magnitudes, it is made evident that granite releases the highest energy, and basalt is second with close volume. The maximum energy is 1010 orders of magnitude for both. Marble releases the lowest energy of a possible maximum energy of Error! Reference source not found.109 orders of magnitude. During the rock burst ejection occurs on the inner wall of the granite sample, while the basalt holes outspread faster, because of the crack propagation. The particle ejection is more active and the rock burst phenomenon is more obvious. Basalt is an extrusive magmatic rock, formed when the magma surface has been cooled and solidified, with a compact structure, and small mineral crystal particles, neat particles, small pores with relatively close contact between particles, and more uniform particle distribution. Granite, however, is an intrusive magmatic rock. Magma intruded along rock fissures, below the surface under high temperature and high pressure conditions, and then became a condensate of igneous rock. Granite is plutonic rock, often formed with well-developed mineral crystal particles. Its mineral crystals are close in contact and texture. Therefore, basalt and granite's peak intensities are higher. Marble occurs under the effects of the earth's internal force during the formation of metamorphic rock, and is composed primarily of carbonate. Its mineral composition is simple, and the connection between mineral particles is loose, making it not sensitive to stress.

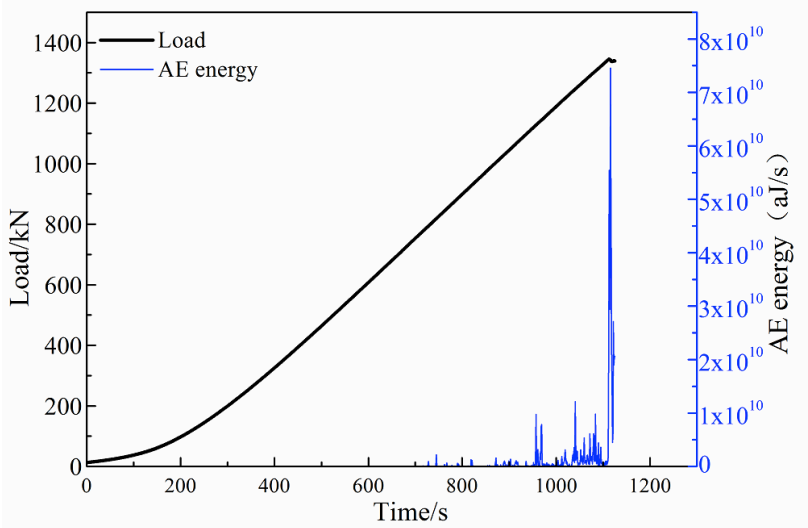

(a)

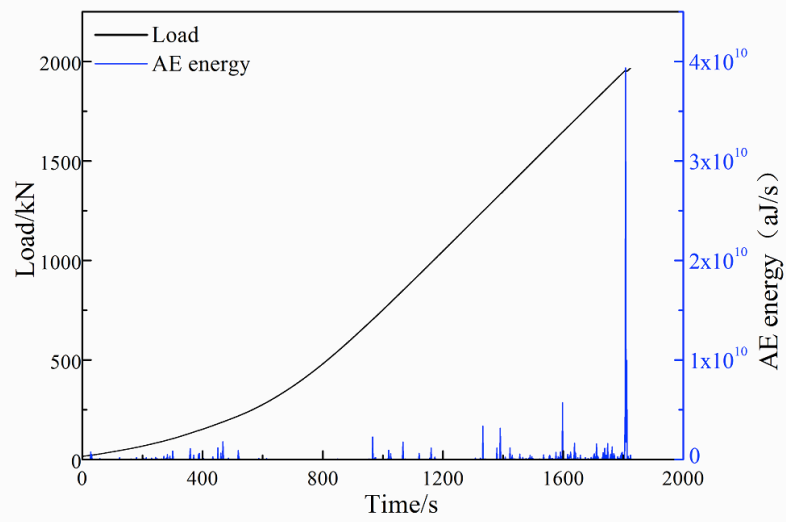

(b)

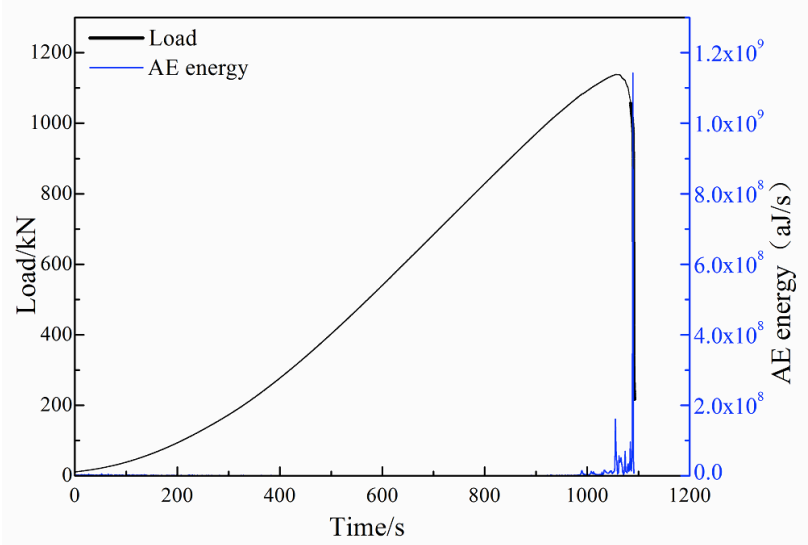

(c)

Fig.6. Curves of load and AE energy. (a) Granite. (b) Basalt.(c) Marble 


\subsubsection{Rock burst process heterogeneity analysis of an $\mathrm{AE}$} event

To describe the heterogeneity of the time and space distribution of earthquake precursors, this document provides a definition of heterogeneity $--\mathrm{C}_{v}$ value [20], referred to as a variation coefficient and applied to describe the cluster of earthquake precursors' anomalies in term of time, and reflected the uneven distribution feature of precursor message in terms of time and space. Accurate descriptions of acoustic emissions distributed in time and space, and understanding its mechanism, can be used to study the uneven degree of the early anomalies preceding a rock blowout. This has great importance in identifying precursors to a rock burst. Sigma and the average value are used in calculation, as follows:

$$
\begin{aligned}
& C_{v}=\frac{S}{\bar{X}} \\
& \bar{X}=\frac{1}{n} \sum_{i=1}^{n} x_{i} \\
& S=\sqrt{\frac{1}{n-1} \sum_{i=1}^{n}\left(x_{i}-\bar{X}\right)^{2}}
\end{aligned}
$$

$n$ as acoustic emission events; $\bar{X}$ as the average of data sequence; $S$ as the data sample standard deviation.

Based on the data collected during three samples' rock burst processes, and the above-mentioned calculation method, $\mathrm{C}_{v}$ value can explode the non-uniform degree of acoustic emission abnormal distribution in the rock burst process, as seen in Figure 7.

Due to the inhomogeneity of the internal medium and the compression strength of the rock, and deformation of each point of nonlinear interaction in the rock, the micro fractures demonstrate inhomogeneity; therefore the common anomaly characteristics will also show obvious heterogeneity. Acoustic emission events are closely related to the crack extensions and the heterogeneity index $\mathrm{C}_{v}$ value of acoustic emission events reflect the non-uniform degree of distribution in acoustic emission activities. Consequently, that index reflects the heterogeneity of the rock acoustic emission events during crack propagation. The analysis in diagram 7 shows that while the load is low, the $C_{v}$ value is low, and the acoustic emission events are more uniformly randomly distributed, reflecting that the randomness of the internal micro crack distribution is stronger. During peak loads to over $80 \%$ of the time, as the increasing of load $\mathrm{C}_{v}$ value rises very slowly, there is a basic-level stage, showing that the acoustic emission event group anomaly is not obvious. Before the rock burst $\mathrm{C}_{v}$ value rise rapidly and significantly, showing an abruptly increasing trend in group abnormalities. The acceleration of the $\mathrm{C}_{v}$ value indicates that large ruptures or cut-through cracks appear partly inside the sample; with more concentrated crack extensions the distribution of acoustic emission events strengthens in partial centralization. This causes an increasing trend in acoustic emission events transforming from a uniform random distribution to a non-uniform one, meaning the heterogeneity index increases sharply.

Prior to the main burst in the three kinds of rock, the $\mathrm{C}_{v}$ value increased differently from the stationary phase to accelerated stage. Granite and basalt's $\mathrm{C}_{v}$ values increased a small range, about 0.2-0.4, while the marble's $\mathrm{C}_{v}$ value increased higher, about 1-1.2, nearly a vertical rising. Marble's acoustic emission event non-uniform distribution showed the biggest change. Prior to the rock burst, the random distribution of internal cracks in the marble was relatively uniform, with no large concentration of cracks. When rock burst occurred, the sample of the cave walls nearly ejected at the same time, and the random distribution cracks expanded quickly, causing a sudden concentration of acoustic emission events, and an abrupt increase in the nonuniform distribution, so the $\mathrm{C}_{v}$ value increased greatly.

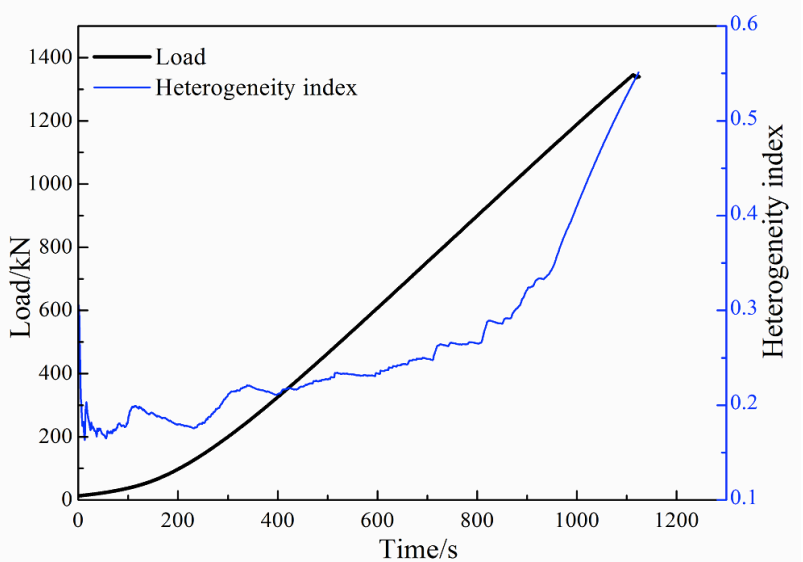

(a)

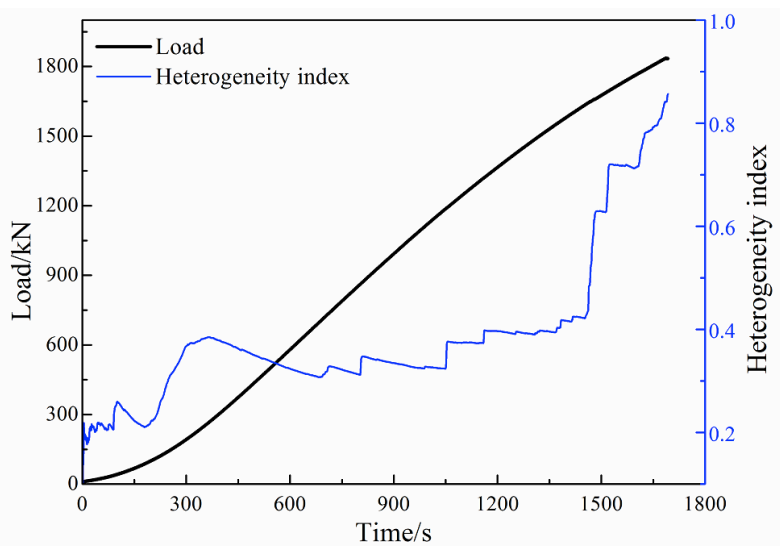

(b)

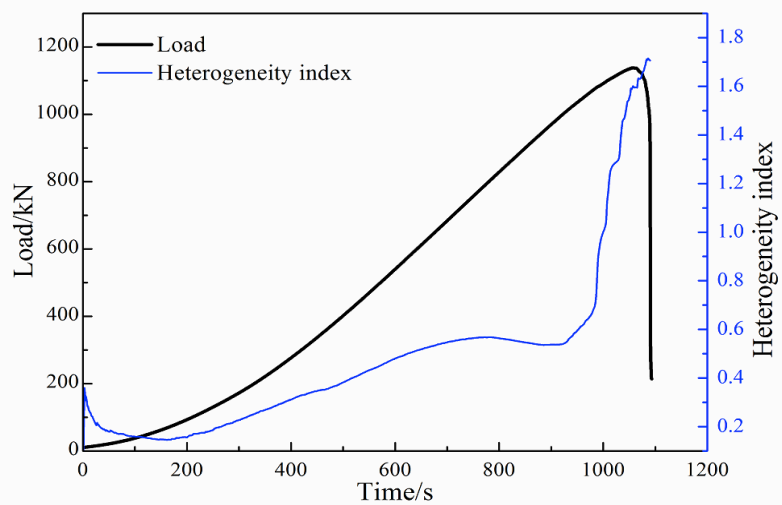

(c)

Fig.7. Curves of load and heterogeneity index. (a) Granite.(b) Basalt.(c) Marble 
The force transmission speed and mineral deformation is different for different rocks, and is bound to cause uneven distributions of inner stress fields in the rock, concentrations of local stress, and micro cracks that result at the weakest part of cementing. Stress concentrations and crack propagations are different in parts; so that an uneven distribution of acoustic emission events appears. In a uniform medium and a non-uniform medium, the rupture occurrence processes are different. Producing a break is abrupt in a homogeneous medium, while in an inhomogeneous medium like rocks, precursory phenomena can be observed before the main rupture occurs. The heterogeneity index $\mathrm{C}_{v}$ value rapidly rises from the stationary phase, predicting that the main rupture inside the rock will form soon, which can be treated as a precursor characteristic of a rock burst occurrence.

\section{Conclusion}

By analyzing the characteristic parameters, this study investigated the effects of various lithology on acoustic emission through rock burst simulation experiments on granite, basalt, and marble, and it can be concluded that:

First, AE events occur more frequently during the rock burst process in granite and basalt. Marble remains dormant within the $75 \%$ of loading time until the peak, at which point its cracks develop rapidly and $\mathrm{AE}$ events increase dramatically and presented obvious periodical characteristics.

Second, during the rock burst process, the AE energy release expresses in group characteristics, which is low energy is released during the incubation phase and robust energy is released during the late phase. And three types of rocks have different performances when releasing energy, with an order of energy releases rate is: granite $>$ basalt $>$ marble.

Third, before a rock burst occurs, there is an increase in the heterogeneity index $\mathrm{C}_{v}$ value of an $\mathrm{AE}$ event which is subject to lithology. The $\mathrm{C}_{v}$ value of granite and basalt has a low increase, about 0.2-0.4, while marble's value is larger, about 1.0-1.2.

In addition, the heterogeneity index $\mathrm{C}_{v}$ value of an $\mathrm{AE}$ event is in line with the rock burst process. Its "stableacceleration" model means an abrupt boost in the nonhomogeneity of an AE event, and the rock burst subject has entered the stage of non-homogeneous crack expansion, which can serve as a precursor of an imminent rock burst.

\section{Acknowledgements}

This work was supported by the National Natural Science Foundation of China under the project No.51374088.

\section{References}

1. Gao, Y. T., Zhou, X. G., and Wu, S. CH., "Simulation experimenta investigation on rock burst of dolomite specimens at great depth tunnels", Journal of University of Science and Technology, 32(7), 2010, pp. 819-826.

2. Tan, Y. A., "The character of rock burst and the structure effect of rock mass", Science in China(Series B), 9(9),1991. pp. 985-991.

3. Xu, D. Q., Shan, X. Y., and Zhang, Y. B., "Using rock acoustic emission and damage analysis of the mechanism of rock burst", Nonferrous Metals (Mining), 21(03), 2000, pp. 28-30.

4. He, M. C., Zhao, F., Zhang, Y., Du, S., and Guan, L. , "Feature evolution of dominant frequency components in acoustic emissions of instantaneous strain-type granitic rock burst simulation tests", Rock and Soil Mechanics, 36(1),2015, pp.1-8.

5. Miao, J. L., He, M. C., and Li, D. J. , "Acoustic emission characteristics of granite under strain rock burst test and its microfracture mechanism", Chinese Journal of Rock Mechanics and Engineering, 28(8), 2009, pp.1593-1603.

6. Zhang, Y. B., Liu, X. X., and Liang, Z. Z., "Experimental study of rock burst precursor of granite tunnel based on multi-physical field parameters", Chinese Journal of Rock Mechanics and Engineering, 33(7),2014, pp.1347-1357.

7. Li, J., Zhang, Y. B., and Liu, X. X., "Experimental Study of Simulated Acoustic Emission of Granites Rock burst under Different Moisture State”, Metal Mine, 454(04), 2014,pp.53-59.

8. Yuan, Z. Q., Tang, L. Z., and Yang, X. C., "Experiment Research on Acoustic Emission Characteristics of Burst-Prone Rocks", Metal Mine, 385(07),2008, pp.132-135.

9. Backers, T., Stanchits, S., and Dresen,G., "Tensile fracture propagation and acoustic emission activity in sandstone: the effect of loading rate", International Journal of Rock Mechanics and Mining Science, 42(7),2005,pp.1094-1101.

10. Cai, M. "Influence of stress path on tunnel excavation responsenumerical tool selection and modeling strategy", Tunnelling Underground Space Technology, 23(6), 2008, pp.618-28.
11. Hiroyuki, S., Sumihiko, M., Tsuyoshi, I., "The distinct element analysis for hydraulic fracturing in hard rock considering fluid viscosity and particle size distributi", International Journal of Rock Mechanics and Mining Sciences, 48(5), 2011, pp.712-727.

12. Luke, P., Frash, Marte, G., Jesse, H., and John, H., "Laboratory simulation of binary and triple well EGS in large granite blocks using AE events for drilling guidance", Geothermics, 55(2), 2015, pp.1-15.

13. Tang, S.F., Tong, M. M., Hu, J. L., He, X. M., "Characteristics of acoustic emission signals in damp cracking coal rocks", Mining Science and Technology (China), 20(1), 2010, pp.143-147.

14. Wang, T., Jiang, Y. D., Zhan, J. J., Wang, C., "Frictional sliding tests on combined coal-rock samples", Journal of Rock Mechanics and Geotechnical Engineering, 6(3),2014, pp. 280-286.

15. Cai, M., Kaiser, PK., Morioka, H., Minami, M., Maejima, T., and Tasaka, Y., et al., "FLAC/PFC coupled numerical simulation of AE in large-scale underground excavations", International Journal of Rock Mechanics and Mining Science, 44(4), 2007, pp.550-64.

16. Jiang, J. H., Guo, Q. L., and Zhou, C. B., "Research on AE characteristics of marble undercompression stress", China Hydraulic Electric Rural Areas, 10(6), 2005, pp.72-4.

17. Jia, R.S., Sun, H.M., and Yan, X.H., "Model for safety evaluation of coal mine roof based on evidence fusion theory", China Coal Sciences 35(9),2010, pp.1496-500.

18. He, M. C., Miao, J. L., and LI, D. J., "Experimental study of rockburst processes of granite specimen at great depth", Chinese Journal of Rock Mechanics and Engineering, 26(5),2007, pp.865876.

19. Hugo, L. C., Mario, G., Elia, M.A., J. C. A, and Wilfrido, M., "Prediction of the Static Modulus of Elasticity Using Four non Destructive Testing", Revista de la Construcción, 13(1), 2014, pp.33-40.

20. Cao, F. J., Jiao, M. R., Wang, H. Y., Zhai, L. N., and Yin, D. L., "Spatial Centrality and Inhomogeneity of Precursory Anomalies in Liaoning region", China Earthquake, 33(1), 2013, pp.127-140. 